
\title{
25 Research Soure \\ DUB1 Suppresses Hippo Signaling Via Modulating TAZ Protein in Gastric Cancer
}

Dehai Wang

Shandong University

Zhongbo Li

Xinxiang medical university

Xin Li

xinxiang medical university

Cheng Yan

Xinxiang University

Huijie Yang

Xinxiang medical University

Ting Zhuang

xinxiang medical university

Xiao Wang

shandong university

Yifeng Zang

shandong university

Ziping Liu

shandong university

Tianshi Wang

shandong university

Ruixia Jia

Shandong University

Peng Su

shandong university

Jian Zhu ( $\sim$ zhujian1204@yahoo.com )

Shandong University Cheeloo College of Medicine https://orcid.org/0000-0003-0171-4251

Yinlu Ding

Shandong university

\section{Research Article}

Keywords: DUB1, TAZ, gastric cancer, deubiquitination, stabilize 
Posted Date: January 19th, 2022

DOI: https://doi.org/10.21203/rs.3.rs-1261007/v1

License: (c) (i) This work is licensed under a Creative Commons Attribution 4.0 International License. Read Full License 


\section{Abstract \\ Background}

The Hippo pathway functions as a tumor-suppressor pathway in human cancers, while the dys-function of Hippo pathway is frequently observed in malignancies. Although the YAP/TAZ activity is tightly controlled by the phosphorylation cascade of MST-LATS-YAP/TAZ axis, it is still unclear why YAP/TAZ protein is activated in human cancers, even Hippo pathway is still active. Besides phosphorylation, recent studies implicate that several post-translational modifications also play critical roles in modulating TAZ function, including ubiquitination.

\section{Methods}

We work on the ER alpha positive breast cancer cell lines and performed western blots, real-time PCR, immuno-precipitation assay, in vitro ubiquitin assay and xenograft mice studies

\section{Results}

Here, by a DUB (Deubiquitinases) siRNA screening library, we discovered DUB1 as a critical modulator to facilitate gastric cancer stemness and progression, which deubiquitinated and activated TAZ protein. We also identified DUB1 was elevated in gastric cancer, which correlated with TAZ activation and poor survival. DUB1 associated with TAZ protein and deubiquitinated TAZ at several lysine sites, which subsequently stabilized and facilitated TAZ function.

\section{Conclusions}

Our study revealed a novel deubiquitinase of Hippo/TAZ axis and one possible therapeutic target for Hippo-driven gastric cancer.

\section{Highlights}

1. DUB1 is an important modulator for Hippo signaling in gastric cancer.

2. DUB1 is elevated in human breast cancer samples and correlates with poor survival in gastric cancer.

3. DUB1 facilitates gastric cancer progression via Hippo/TAZ axis.

4. DUB1 associates with TAZ and directly inhibits P53 poly-ubiquitination and degradation.

\section{Background}


Gastric cancer is the fifth leading incidence and the fourth cancer-related mortality in China[1]. According to the epidemiology reports in 2020, more than one million new gastric cancer cases were diagnosed, while more than half of the cases were occurred in China[2]. The carcinogenic process of gastric cancer is the multi-step revolutions of several gastric lesions, including gastritis, intestinal metaplasia and epithelial neoplasia[3]. Due to insidious onset and minor symptoms, most of the gastric cancer patients are diagnosed at advanced stage with the 5-year survival rate only 35\%[4]. Several biomedical studies showed the involvement of Helicobacter pylori infection and chronic inflammations for gastric cancer development[5], however the detailed mechanisms for gastric cancer progression are still unclear. Thus, it is urgent and necessary to develop novel biomarkers and therapeutic targets for gastric cancer treatments.

Several studies have built the potential link between Hippo signaling and gastric cancer[6]. Hippo signaling pathway plays essential roles in several biological processes, including organ size control, tissue homeostasis, carcinogenesis and immune response[7]. The activation of Hippo signaling is subject to a phosphorylation cascade, in which the mammalian Hippo kinases MST1/2 phosphorylates the LATS1/2 kinase, while the phosphorylated LATS1/2 promotes the phosphorylation of YAP/TAZ protein, leading the localization in the cytosol and protein degradation[8]. If the Hippo signaling is inactivated, the YAP/TAZ are unphosphorylated, translocate into the nuclear and interact with several transcriptional factors to promote their target genes expression[9]. The dys-regulation of Hippo signaling was observed in several human malignancies. While the over-expression of YAP/TAZ was found in gastric cancer, the blockage of YAP/TAZ function could inhibit gastric cancer progression in H.pylori-induced mice models[10]. However, since a lot of efforts have been exerts to pharmaceutically target Hippo signaling, the clinical application is still pre-mature.

TAZ (Transcriptional Co-Activator With PDZ-Binding Motif) is a downstream effector of Hippo signaling and a transcriptional co-factor for several transcription factors, including TEADs[11]. Several studies implicated that TAZ could be a critical factor for carcinogenesis in human cancers. For example, Molecular studies revealed that TAZ activation could trans-activate several signaling pathways, such as Wnt and Hedgehog signaling[12, 13]. In human cancer samples, TAZ protein level was elevated in server cancers, including breast cancer and gastric cancer and its expression level was correlated with poor prognosis[14-16]. Based on this, target TAZ could be a promising strategy, which subsequently blocks its trans-activation effects. Thus, as the obvious candidate component of ubiquitin-proteasome system, target TAZ protein stability could be utilized to rescue tumor-suppression function of Hippo signaling and gastric cancer progression.

The regulation of protein stability is subject to the balance of E3 ubiquitin ligases and deubiquitinases, which is crucial to maintain normal physiological homeostasis[17, 18]. The disrupted regulation of protein ubiquitination could contribute to several human diseases, including cancers[19]. We and other groups discovered several E3 ubiquitin ligases, which exerted their effects on Hippo pathway effectors and modulated Hippo signaling activity and cancer progression[20-25]. Although there are studies reporting the involvement of several deubiquitinases in modulating Hippo signaling, it is still largely 
unclear which of the deubiquitinases exerting important impacts on Hippo signaling. By a DUB (Deubiquitinases) siRNA screening library, we identified DUB1 as a critical modulator in modulating Hippo pathway activity in gastric cancer. DUB1 was increased in gastric cancer samples, correlated with poor prognosis in gastric cancer patients and correlated to TAZ expression. DUB1 facilitated gastric cancer progression through Hippo/TAZ axis. DUB1 was found to associate with TAZ in the nuclear and inhibited K48-linked ubiquitination and degradation. Hence, DUB1 functions to strengthen Hippo signaling activity in gastric cancer, which could be a promising marker for gastric cancer diagnostics and therapeutics.

\section{Materials And Methods}

\section{Cell culture}

The gastric cancer cell lines (AGS and MGC803) were cultured in DMEM Medium (ThermoFisher Scientific, Cat: 11965092) containing 10\% fetal bovine serum (Biological Industries, BISH0744), 1\% penicillin/streptomycin (Invitrogen). HEK-293 cells were cultured in DMEM Medium supplemented with 10\% FBS (Biological Industries, BISH0744) and 1\%penicillin/streptomycin. The gastric cancer AGS and BCG803 cells were authenticated by short tandem repeats profiling (STR). STR profiling of our AGS cells was found to be $100 \%$ consistent with the STR data of the AGS from China Infrastructure of Cell Line Resources. MGC803 cell STR profiling data was not accessible in public databases including ATCC. Cells were regularly tested for mycoplasma using Lookout Mycoplasma PCR detection kit (MP0035, Sigma) and only used when negative.

\section{RNA isolation and quantitative real-time PCR (qRT-PCR)}

Total RNA was extracted with RNeasy Plus Mini Kit (Tiangen, DP451) following the manufacturer's specifications. Reverse transcription was performed using the HiScript II Q RT SuperMix (Vazyme, R22301). qRT-PCR was carried out using SYBR qPCR Master Mix (Vazyme, Q511-02) and 7500 Fast Real-Time PCR System (Applied Biosystems, Singapore). The 36B4 was used as an internal control. The sequence of the primers for qPCR was listed as follows: 36B4 F: GGC GAC CTG GAA GTC CAA CT; R: CCA TCA GCA CCA CAG CCT TC. CTGF F: CTC GCG GCT TAC CGA CTG; R: GGC TCT GCT TCT CTA GCC TG. CYR61 F: AGC AGC CTG AAA AAG GGC AA; R: AGC CTG TAG AAG GGA AAC GC. DUB1 F: GAG GCC GGG GCT CTG A; R: ACT GGG ATG TGC AGA CTT GG. TAZ F: AGA GTC GGG TCG GGA TTT GT; R: AGG CCG GAT TCA TCT TCT GGG

\section{Plasmids and siRNA}

The DUB1 and TAZ plasmid are acquired from HANBIO Company (https://www.hanbio.net). The DUB1 and TAZ deletion constructs were sub-cloned from the full-length plasmid. The HA-K48 and HA-Ub plasmids were used in previous study.. The Lipofectamin 2000 (1662298, Invitrogen) was used for the plasmids transfection. Small interfering RNAs were used for specific gene knocking-down. The DUB1 siRNA sequences were: (1) CCG GCA AGC UGC GAA UAU UTT; AAU AUU CGC AGC UUG CCG GTT, and (2) GCA CAC CAC UGA AGA GAU UTT; AAU CUC UUC AGU GGU GUG CTT. The negative control siRNA 
sequences were: UUC UCC GAA CGU GUC ACG UTT; ACG UGA CAC GUU CGG AGA ATT. The RNA iMAX reagent (13778150, invitrogen) was used for siRNA transfection. For lenti-virus based DUB1 silencing, shDUB1 was cloned into the vector pLK0.1, which was co-transfected with pMD2.G and psPAX2 envelop plasmids into HEK293 cells. The DUB1 shRNA lenti-virus was harvested after 48 hours. The gastric cancer cells were incubated with $2 \mathrm{ml}$ antibiotic-free medium containing 200 ul lentivirus.

\section{Western blot}

Standard western-blot assays were utilized to analyze the relative protein expression in cells. The following antibodies were used for western blot assays: anti-Flag-M2 (A8592, Sigma, 1:1000), anti-HA (2013819001, Roche, 1:1000) anti-Myc (9E10, Santa Cruz, 1:1000), anti-GAPDH (0411, Santa Cruz, 1:1000), anti-TAZ (Cell Signaling Technology, CST83699, 1:1000), anti-DUB1 (Sigma, HPA12082, 1:1000), Protein signals were detected with an ECL kit (Millipore Co., Billerica, Massachusetts, USA).

\section{Luciferase reporter assays}

For TEAD luciferase activity assays, the MGC803 and AGS with siDUB1 or siControl cells transfected with the TEAD luciferase reporter vector for $24 \mathrm{~h}$. Cells were then harvested for assays. Luciferase reporter assays were performed using the dual luciferase assay kit (Promega). The pRL-null vector expressing renilla luciferase (Promega) was used as an internal control to normalize the transfection efficiency.

\section{Wound healing and Transwell assays}

For the wound-healing assay, the MGC803 and AGS with siDUB1 or siControl cells were seeded in a 6-well plate until confluent and then wounded with a sterile tip. The cells pictures were acquired at the indicated time points after scratching. The distances between the two edges of the scratched wound were measured using Image J software. The trans-well system ( $8 \mu \mathrm{m}$ pore size, Corning) was employed for cell migration and invasion assays. For invasion assays, the upper chambers were coated with matrigel (BD Biocoat, USA). After $24 \mathrm{~h}$, the gastric cancer cells that had migrated through to the bottom of the insert membrane were fixed, stained with crystal violet and counted under $\times 20$ objective lens. The experiments were in three repeats.

\section{Cycloheximide assay}

MGC803 cells were transfected with siRNA or siControl for 24 hours. After that, the Cycloheximide was added into culture medium with the final concentration of $100 \mu \mathrm{mol} / \mathrm{L}$. Cell lysis were collected at 0, 3, 6 and 9 hours after the treatment of cycloheximide. For HEK293 cells, the cells were transfected with 2 ug Flag-DUB1 or Flag vector. After 24 hours, cells were treated with cycloheximide with final concentration of $100 \mu \mathrm{mol} / \mathrm{L}$. Cell lysis were collected at 0, 3, 6 and 9 hours after the treatment of cycloheximide.

\section{Immunofluorescence (IF) staining}


The MGC803 Cells on the coverslips were fixed with $4 \%$ paraformaldehyde and incubated with the primary antibody against DUB1 (Sigma, A300-940A), TAZ (CST, 71192) at $4{ }^{\circ} \mathrm{C}$ overnight. After that, the cells were washing with PBS. Then the cells were then incubated together with fluorescence-conjugated secondary antibody (Invitrogen, Carlsbad, CA). Finally, the cells were subsequently counterstained with DAPI (Life Technology). The staining Images were captured through the confocal laser-scanning microscope (Leica TCS SP8 STED). The fluorescence-integrated density was measured by ImageJ software.

\section{Clone formation assays}

The MGC803 and AGS were seeded in six-well plates overnight and treated with 50nM DUB1 siRNA or 50nM siControl. After twenty-four hours, the gastric cancer cells were washed with PBS, trypsinized and plated at low density (5000 cell/well in six-well plate). The cells were cultured for 10 days and the medium was refreshed every two or three days. The colonies were stained with crystal violet. The number of the clones in a given area was counted for each condition.

\section{Co-IP assays}

The co-immunoprecipitation assays were performed as previous described. The MGC803 total cell lysls were collected and pre-cleared with rabbit lgG for $2 \mathrm{~h}$ and subsequently immunoprecipitated with DUB1 antibody (Sigma, A300-940A) over night, while rabbit IgG (Santa Cruz) was used as the negative control. The bounded protein was analyzed by Anti-TAZ (CST, 71192). For the overexpression experiment, HEK293 cells were transfected with 5ug Flag-DUB1 (Full length or deletion domains) and Myc-TAZ (Full length or deletion domains) in $10 \mathrm{~cm}$ dish. Cell lysates were pre-cleared with IgG and subsequently incubate with anti-Flag-M2 (A8592, Sigma, 1:1000) antibody, while mouse lgG was used as the negative control. The bound proteins were analyzed by western blotting.

\section{In vivo ubiquitination assays}

For in vivo ubiquitination assays, cells were transfected with vectors, including expressing Myc-TAZ, FlagDUB1 and HA-Ub for $24 \mathrm{~h}$ respectively. Cells were then treated with MG132 $(10 \mu \mathrm{M})$ for $6 \mathrm{~h}$, and the levels of Myc-TAZ ubiquitination was determined by IP with anti-HA antibody (2013819001, Roche,1:1000) followed by western-blot assays with an anti-Myc (9E10, Santa Cruz, 1:1000).

\section{In vivo tumorigenesis assay}

For in vivo tumorigenic experiment, MGC803 cells were infected with shControl virus or shDUB1 virus. After 48 hours of infection, cells were treated with $1 \mathrm{ug} / \mathrm{ml}$ puromycin for 3 days. MGC803 cells $\left(2 \times 10^{6}\right)$ were injected into the right dorsal flank of 4-week-old female BALB/c nude mice. Tumor formation in nude mice was monitored over a 4-week period. The tumor volume was calculated by the formula: tumor volume $=0.5 \times$ length $\times$ width $^{2}$. The mice were sacrificed five weeks after injection. The mice were 
sacrificed and the tumors were weighted and photographed. The experiments were performed under the protocols approved by ethnic committee of Xinxiang Medical University.

\section{Cell proliferation assay}

MGC803 and AGS cells were transfected with siDUB1 or siControl in 24-well plates. Twenty-four hours after transfection, the cell number was countered and 4000 cells were seeded into 96 -well plates. The relative cell viability was measured at the indicated time points. Cell numbers determined determined using CCK8 cell proliferation reagent by measuring the absorbance at $450 \mathrm{~nm}$. Cell proliferation was further analyzed by EdU incorporation and flow cytometry. Gastric cancer cells were determined by using the 5-ethynyl-20-deoxyuridine (EdU) assay kit (Ribobio, Guangzhou, China). For quantification analysis of the images, each data point represents the positive fluorescence area calculated from a minimum of five randomly chosen fields from three individual experiments. EdU incorporation FACS assay was performed according to the manufacturer's instructions. The experiments were performed in triplicate. For cell cycle analysis, MGC823 cells were transfected with $50 \mathrm{nM}$ siDUB1 or siControl. After 24 hours, the cells were fixed with $70 \%$ ethanol and stained with propidium iodide. Twenty-four hours post transfection, the cells were stained with propidium iodide and annexin V. A BD LSR FACS was used to measure the fluorescence intensity.

\section{Tissue microarray (TMA) and immunohistochemistry (IHC)}

One hundred paraffin-embedded gastric cancer samples were acquired from Shanhai OTUDO Biotech Company (http://www.superchip.com.cn). All the gastric tumor samples were examined with pathological specialists. The pathological grade plus lymph node metastasis status of each sample were acquired from Shanhai OTUDO Biotech Company. The usage of the samples was approved from the Shanhai OTUDO Biotech Company with written informed consent from all the patients. Specific antibodies against TAZ (CST, 71192) and DUB1 (Sigma, HPA12082) were used to detect the staining density in human samples. The scores were calculated on the intensity and percentage of positive tumor cells in the whole tissue, which were evaluated according to the Fromowitz Standard. The staining intensity was graded as: no staining, 0 ; weakly positive, 1 ; moderately positive, 2 and strongly positive, 3 . The percentage for positive cells was into four grades: $0-25 \%$ staining, $1 ; 26-50 \%$ staining, $2 ; 51-75 \%$ staining, 3 and $76-100 \%$ staining, 4 . The staining 1-2 was regarded as low expression, while the stain 3-4 was regarded as high expression. All staining were assessed at 200X magnifications and at least three fields from each core were counted.

\section{RNA sequencing and data analysis}

The global gene expression analysis (siControl and siDUB1) was based on RNA sequencing platform from BGI (Beijing Genomic Institute). The RNA sequence data are deposited in the Gene Expression Omnibus (GEO) database (Assessing number: GSE143947). Analysis was performed for differentially expressed genes $(P<0.01$ and fold change $>2)$ by Ingenuity Pathway Analysis (IPA). For gene set enrichment analysis of RNA-seq data, gene sets of Conserved Hippo Signature were used and 
downloaded from Molecular Signatures Database v7.4, GSEA was implemented using the GSEA 4.1.0 software, with default parameters. Volcano plot was generated using 'ggplot2' package in R (threshold $\mathrm{P}<0.05$ and fold change $>1.5$ ).

\section{Analysis of TCGA data and Progression-free survival data analysis}

Gene expression data for 385 TCGA gastric cancer patients were downloaded from the webpage (http://gepia.cancer-pku.cn/index.html). The expression of DUB1 mRNA level between normal gastric tissue and different gastric cancer stages were generated from GEPIA online software. The progressionfree survival (PFS) survival data of TAZ and DUB1 were generated from KMPLOT online analysis database (https://kmplot.com). The gene affy ID was 202133_at for TAZ and 227093_at for DUB1. The PFS survival data of DUB1 and TAZ in gastric cancer patients were generated from KMPLOT database.

\section{Statistical analysis}

No specific statistical tests were used to predetermine the sample size. Statistical analysis was performed using GraphPad Prism 7 software or SPSS version 23.0. Data are expressed as the mean \pm s.e.m. values. Differences between two independent groups were evaluated with Student's t-test. The Kaplan-Meier method with the log-rank test was applied for survival analysis. Differences were considered to be statistically significant when $P<0.05$ ( ${ }^{\star} P<0.01$; $\left.* * P<0.001\right)$.

\section{Results}

\section{DUB1 is elevated in human gastric cancer and correlates with Hippo signaling activity in whole genomic scale}

Since this studies aimed to identify novel de-ubiquitinating enzymes in regulation Hippo signaling, we carried out a DUB (Deubiquitinases) siRNA screening via the DUB siRNA library (Dharmacon Company, Cat: G104705). Since HEK293 cells were widely used for Hippo signaling study and could be transfected with high efficiency, we utilized HEK293 cells for initial screening. Since CTGF was regarded as one of the most classical target genes, we used CTGF as the endpoint to indicate Hippo signaling activity (Fig. 1A). The siRNA screening coupled with QPCR revealed several confirmed DUBs, such as USP9X and USP7, which were proved to regulate Hippo pathway in previous publications (Supplementary Table 1). However, we discovered an unreported deubiquitinase-DUB1 could also be an important regulator in Hippo pathway (Fig. 1B). We further analyzed the DUB1 mRNA level in gastric cancer patients. The TCGA data showed that DUB1 expression was significantly elevated in gastric cancer samples compared with normal gastric tissue (Fig. 1C). The immunohistochemistry data showed that DUB1 was significantly higher in gastric cancer samples, which expression was correlated with lymph node metastasis and later clinical stage $(P<0.001 ; P<0.001, P<0.001$ respectively; Fig. 1D -1E). Interestingly, the protein level of DUB1 positively correlated with TAZ expression in gastric cancer samples $(P<0.001$, Fig. 1F). The prognostic data analysis showed that both DUB1 and TAZ correlated with poor survival in gastric cancer patients (Fig. 1G-1H). In order to approach the function of DUB1 in gastric cancer in an unbiased way, we deplete DUB1 in 
MGC803 cells for the whole genomic expression analysis. The KEGG pathway analysis indicated that DUB1 depletion could affect several cancer-related pathways, including Hippo signaling and PI3K/AKT pathway (Fig. 1I). The GSEA analysis implicated that DUB1 depletion significantly inhibited Hippo signaling activity (Fig. 1J). The volcano plot analysis showed DUB1 silencing dramatically inhibited Hippo classical target gene expression, including CTGF, CYR61 and ANKRD1 (Fig. 1K).

\section{DUB1 facilitates Hippo/TAZ axis in gastric cancer cells}

We further utilized two independent siRNAs for DUB1 to avoid off-target effects. The western blotting assays showed that DUB1 depletion could not affect YAP protein level in AGS and MGC803 cells (Data not shown). However, DUB1 depletion could dramatically decrease TAZ protein level in AGS and MGC803 cells (Fig. 2A-2B). We examined the Hippo target gene expression in gastric cancer cells. We found that DUB1 depletion could inhibit Hippo target gene expression, including CTGF and CYR61, in AGS and MGC803 cells (Fig. 2C-2D). We further evaluated the effect of DUB1 in Hippo signaling activity. The luciferase reporter assay showed that DUB1 silencing inhibited TEAD response element activity in AGS and MGC803 cells (Fig. 2E-2F). Consistently, the transient DUB1 overexpression in HEK293 cells showed increased TAZ protein level, Hippo target gene expression (CTGF and CYR61) and the increased luciferase activity of TEAD response elements (Fig. 2G-2I).

\section{DUB1 depletion inhibits gastric cancer progression in vivo and in vitro.}

In order to investigate the impact of DUB1 in gastric cancer phenotype, we depleted DUB1 in MGC803 and AGS cells. The CCK8 assay indicated that DUB1 depletion significantly inhibited gastric cancer cell growth (Fig. 3A-3B). Besides, The EdU incorporation assays showed that DUB1 silencing could dramatically decrease the EdU positive cells in MGC803 and AGS cells (Fig. 3C-3D). The cell cycle analysis by PI staining and FACS analysis showed that DUB1 depletion significantly caused the increased proportion of G1 phase cells (Fig. 3E-3F). Besides, the trans-well assay implicated that DUB1 was necessary for gastric cancer invasion capacity in AGS and MGC803 cells (Fig. 3G-3H). The woundhealing assay showed that DUB1 depletion could decrease the cancer cell migration speed in AGS and MGC803 cells (Fig. 3I-3J). In the in vivo tumor growth assay, stable silencing of DUB1 in MG803 cells could inhibit tumor growth in the xenograft mice model (Fig. 3K-3M).

\section{DUB1 controls gastric cancer progression via Hippo/TAZ axis}

In order to investigate the logic link between the gastric cancer phenotype and Hippo/TAZ signaling in DUB1 function, we carried out several rescue experiments. DUB1 depletion could decrease TAZ protein level, which could be rescued by TAZ over-expression in MGC803 and AGS cells (Fig. 4A-4B). The QPCR assay showed that DUB1 silencing could decrease Hippo target gene expression, which could be rescued by TAZ overexpression in MGC803 and AGS cells (Fig. 4C-4D). The luciferase reporter assay showed that DUB1 silencing inhibited TEAD response element activity in AGS and MGC803 cells, which could be rescued by TAZ overexpression (Fig. 4E-4F). The CCK8 assay showed that the inhibited cell growth by DUB1 depletion could be rescued by further TAZ overexpression in MGC803 and AGS cells (Fig. 4G-4H). 
The EdU staining assay showed that the TAZ over expression could rescue the EdU positive cells, which was decreased by DUB1 depletion (Fig. 4I-4J). The cell cycle analysis showed that $\mathrm{G} 1$ arrest caused by DUB1 depletion could at least partially rescued by further TAZ overexpression (Fig. 4K-4L). The clone formation assays showed that the decreased clone numbers caused by DUB1 depletion could be rescued by further TAZ overexpression in MGC803 and AGS cells (Fig. 4M). In figure 4N-4O, DUB1 knocking-down in gastric cancer cells could inhibit cell invasion, which effect could be at least partial rescued by further TAZ overexpression. The wound-healing assay showed that DUB1 depletion could inhibit cell migration speed, which effect could be further rescued by TAZ overexpression in MGC803 and AGS cells (Fig. 4P4Q). These data indicated that DUB1 modulated Hippo signaling through TAZ protein in gastric cancer.

\section{DUB1 associates with TAZ and modulates TAZ stability in gastric cancer cells}

We further investigate the localization of DUB1 and TAZ in gastric cancer cells. The immuno-staining shows that both DUB1 and TAZ are mainly localized in the nuclear (Fig. 5A). This data was further confirmed by nuclear cytoplasm separation assay (Fig. 5B). The endogenous immuno-precipitation showed that DUB1 could interact with TAZ in MGC803 cells (Fig. 5C). The DUB1 protein is composed of USP domain (Deubiquitinase domain), central domain and CTD (C-terminal domain) domain, while TAZ protein is composed of TB domain (TEAD binding domain), WW domain and TA domain (Transcription activation domain) (Fig. 5D-5E). We made these deletion constructs and further investigated the associated domain by IP assay. The data showed that WW domain was required for TAZ to interact with DUB1, while the CTD domain of DUB1 was responsible domain for interaction with TAZ (Fig. 5F-5G). DUB1 depletion could decrease TAZ protein level in MGC803 cells, which effects could rescued by MG132 treatments (Fig. 5H). In consistent, transfection of DUB1 in HEK293 cells showed that DUB1 could increase TAZ protein level, which effect could be minimized with the presence of the proteasome inhibitor MG132 (Fig. 5I). These data indicate that DUB1 could modulate TAZ protein level through proteasome degradation system. We further tested the protein stability in HEK293 system, which showed that DUB1 overexpression could enhance TAZ stability (Fig. 5J). This was further confirmed in MGC803 cells via endogenous TAZ depletion (Fig. 5K).

\section{DUB1 stabilizes TAZ via inhibiting TAZ K48-linked poly-ubiquitination.}

Since DUB1 is one Deubiquitinating enzyme, we further investigated the role of DUB1 in TAZ polyubiquitination. The ubiquitination-based immuno-precipitation in HEK293 cells showed that DUB1 overexpression could inhibit TAZ overall poly-ubiquitination (Fig. 6A). Since K48-linked ubiquitination is the most common degradation manner, we examined the DUB1 effect on K48-linked ubiquitination of TAZ. In HEK293 cells, we observed that DUB1 overexpression could inhibit K48-linked ubiquitination of TAZ (Fig. 6B). In the MGC803 cells, we observed that depletion of endogenous DUB1 could enhanced the overall poly-ubiquitination and K48-linked poly-ubiquitination (Fig. 6C-6D). We further investigated the functional domain for DUB1 to exert its function on TAZ ubiquitination. In the ubiquitination-based immuno-precipitation assay, we observed that the USP domain (1-420 AA) of DUB1 was necessary for its deubiquitination function on TAZ protein (Fig. 6E-6F). 


\section{Discussion}

In our study, we discovered a novel deubiquitinase family member DUB1 through DUB library siRNA screening. As an important endogenous modulator for Hippo/TAZ axis in gastric cancer progression, DUB1 was elevated in human gastric cancer and related to poor survival for gastric cancer patients. The IHC analysis showed that DUB1 expression correlated with metastasis and TAZ expression, while DUB1 depletion significantly inhibited Hippo signature genes expression in whole genomic scale. DUB1 inhibition caused cell growth inhibition, decreased cell cycle progression and decreased cell invasion capacity. The molecular biology assays showed that DUB1 could associate with TAZ protein and stabilize TAZ protein via inhibited TAZ K48-linked poly-ubiquitination in gastric cancer (Fig. 6G). Based on these, we can propose inhibition of DUB1 expression or pharmaceutical targeting DUB1 function could be a promising strategy to inhibit the Hippo/TAZ-driven gastric cancers.

The conserved Hippo signaling maintains tissue hemostasis and organ size control[26]. Several studies showed that the dys-regulation of Hippo pathway was also exist in gastric cancer[27-30]. For example, the Hippo pathway effector YAP/TAZ was significantly elevated in human gastric cancer and related to poor overall survival, while depletion of YAP/TAZ or pharmaceutically targeting YAP/TAZ-TEAD interaction could inhibit gastric cancer growth in vitro and in vivo[31]. Based on these facts, we can propose that the dys-function of Hippo signaling could be one of the driver events for gastric cancer. As the "Oncogenic addiction" pathway, targeting Hippo signaling effector YAP/TAZ could a plausible strategy for gastric cancer. On the other hand, as an auto-inhibitory pathway, the control of Hippo pathway activity depends on several internal inhibitors, such as FATs and AJUBA, and functional cascade of MST-LATS-YAP/TAZ, while the abnormality of Hippo signaling could link to several human cancers[32]. Several genetic studies from different malignancies reported that the genetic mutations of Hippo inhibitors, such as FATs and AJUBA, or YAP/TAZ amplifications were commonly observed[32]. However, such genetic events were rarely found in gastric cancer, which might indicate the activation of Hippo effector YAP/TAZ undergoing some unknown mechanisms.

The Hippo signaling effector TAZ plays important roles in controlling Hippo signaling activity, which contains three domains[33]. The TEAD interaction domain is responsible for its association with several transcriptional factors, while the WW domain mainly interacts with several TAZ modulators[34]. Due to the extensive interactive face between TAZ and several transcriptional factors, directly targeting TEADTAZ interaction becomes technically challenging. Based on the fact that TAZ protein is highly active, while the inhibitory factors remains functional in gastric cancer. Our research team starts to target Hippo effectors via modulating protein stability. Through a DUB siRNA library, which contained more than 100 deubiquitinases, we discovered DUB1 as a novel effector for TAZ stability and gastric cancer progression. Such findings identified novel endogenous modulators for Hippo signaling, but also promising targets to Hippo/TAZ in gastric cancer treatments.

Our previous studies characterized several E3 ubiquitin ligases in modulator Hippo signaling effectors and cancer progression, such as RNF187 and ZNF213[20, 24]. However, the protein stability is controlled 
via a balance between ubiquitinations and deubiquitinations. The deubiquitinases, which remove the ubiquitin chains from the target proteins, mainly function to stabilize target proteins[35]. Currently, there are approximately more than 100 DUBs identified in human genomes, while the ubiquitin specific peptidases is the largest group[36]. DUB1 (also known as USP36) gene was firstly discovered from Hela cells in 2004, which subsequently reported to be function as one deubiquitinase through the USP domain[37]. DUB1 was found to modulate RNA helicases stability for RNA transcription in cancer[38]. Besides, DUB1 was also reported to stabilize Myc and facilitate myc-driven cancer progression, while depletion of DUB1 could cause growth inhibition and autophagy through P62/SQSTM1 pathway[39]. Couple with our conclusion that DUB1 stabilizing TAZ in cancer progression, we can propose that targeting DUB1 could block multiple oncogenic pathway and synergize in cancer therapeutics.

\section{Conclusions}

We have discovered DUB1 as an oncogene for gastric cancer both in clinical samples and experimental studies. We demonstrated that DUB1 was elevated in gastric carcinoma and related to poor survival. DUB1 associated with TAZ protein, inhibited TAZ poly-ubiquitination and proteasome-dependent degradation in gastric cancer cells. Our studies revealed a novel function of DUB1 in Hippo signaling in multiple layers. As a novel modulator for Hippo signaling, modulation of DUB1 activity or gene expression level could be an appealing strategy to treat gastric cancer.

\section{Abbreviations}

DUB

deubiqutinase

USP

ubiquitin specific peptidese

TAZ

Transcriptional Co-Activator With PDZ-Binding Motif

PFS

progression-free survival

EdU

5-ethynyl-2'-deoxyuridine

GSEA

gene set enrichment analysis

$\mathrm{IHC}$

Immunohistochemistry

\section{Declarations}

Acknowledgment: We thank all the members of Xinxiang Key Laboratory of tumor migration and invasion precision medicine for sharing valuable material and research support. 


\section{Authors' contributions}

Dehai Wang, Zhongbo Li, Xin Li, Cheng Yan and Huijie Yang performed most of the bench work. Ting Zhuang and Xiao Wang performed the DUBs screening assay. Ziping Liu, Tianshi Wang and Yifeng Zang performed the IHC assay and data analysis. Jian Zhu, Yinlu Ding and Peng Su supervised the process of the study and performed the manuscript writing. All authors read and approve the final manuscript.

Funding: The project was supported by the National Science Foundation for Young Scientists of China (No. 82172999, Ting Zhuang), the Program for Science \& Technology Innovation Talents in Universities of Henan Province (Grant No. 21HASTIT049 to Ting Zhuang), the Taishan Scholar Program of Shandong Province (tsqn202103175 to Jian Zhu), Shandong Provincial National Natural Science Foundation (ZR2016HQ44 to Yinlu Ding; ZR2021MH017 for Jian Zhu) and the Program for Science \& Technology Innovation in Shandong Society of Geriatrics (LKJGG2021z002).

Availability of Supporting Data and Materials: The publicly available data are provided in GEO database. The original siRNA screening data are provided in supplementary materials. The original data for WB and QPCR are provided in supplementary materials. The cell line authentications are shown in supplementary materials. The approved document of human sample ethnics is shown in the supplementary materials.

Ethics Approval and Consent to participate: This study was reviewed and approved by the Ethical Board at The Second Hospital of Shandong University.

Consent for publication: Not applicable.

Competing Interests: There is no competing interest to declaim.

\section{Author details:}

${ }^{1}$ Department of General Surgery, The Second Hospital, Cheeloo College of Medicine, Shandong University, Shandong Province, P.R. China

${ }^{2}$ Xinxiang Key Laboratory of Tumor Migration and Invasion Precision Medicine, School of Laboratory Medicine, Xinxiang Medical University, Xinxiang 453003, Henan Province, P.R. China

${ }^{3}$ School of Medicine, Xinxiang University, Xinxiang, Henan P.R. China

${ }^{4}$ Department of Burns and Plastic Surgery, The Second Hospital, Cheeloo College of Medicine, Shandong University, Shandong Province, P.R. China

${ }^{5}$ Department of Hepatobiliary Surgery, The Second Hospital, Cheeloo College of Medicine, Shandong University, Shandong Province, P.R. China

${ }^{6}$ Department of Pathology, Shandong University Qilu Hospital, Cheeloo College of Medicine, Shandong University, Shandong Province, P.R. China 


\section{References}

1. Sung H, Ferlay J, Siegel RL, Laversanne M, Soerjomataram I, Jemal A, Bray F. Global Cancer Statistics 2020: GLOBOCAN Estimates of Incidence and Mortality Worldwide for 36 Cancers in 185 Countries. CA Cancer J Clin. 2021;71(3):209-49.

2. Rawla P, Barsouk A. Epidemiology of gastric cancer: global trends, risk factors and prevention. Prz Gastroenterol. 2019;14(1):26-38.

3. Correa P. Human gastric carcinogenesis: a multistep and multifactorial process-First American Cancer Society Award Lecture on Cancer Epidemiology and Prevention. Cancer Res. 1992;52(24):6735-40.

4. Chen ZD, Zhang PF, Xi HQ, Wei B, Chen L, Tang Y. Recent Advances in the Diagnosis, Staging, Treatment, and Prognosis of Advanced Gastric Cancer: A Literature Review. Front Med (Lausanne). 2021;8:744839.

5. Polk DB, Peek RM, Jr. Helicobacter pylori: gastric cancer and beyond. Nat Rev Cancer. 2010;10(6):403-14.

6. Kang W, Cheng AS, Yu J, To KF. Emerging role of Hippo pathway in gastric and other gastrointestinal cancers. World J Gastroenterol. 2016;22(3):1279-88.

7. Yu FX, Zhao B, Guan KL. Hippo Pathway in Organ Size Control, Tissue Homeostasis, and Cancer. Cell. 2015;163(4):811-28.

8. Meng Z, Moroishi T, Guan KL. Mechanisms of Hippo pathway regulation. Genes Dev. 2016;30(1):117.

9. Mo JS, Park HW, Guan KL. The Hippo signaling pathway in stem cell biology and cancer. EMBO Rep. 2014;15(6):642-56.

10. Plouffe SW, Hong AW, Guan KL. Disease implications of the Hippo/YAP pathway. Trends Mol Med. 2015;21(4):212-22.

11. Hong W, Guan KL. The YAP and TAZ transcription co-activators: key downstream effectors of the mammalian Hippo pathway. Semin Cell Dev Biol. 2012;23(7):785-93.

12. Azzolin L, Zanconato F, Bresolin S, Forcato M, Basso G, Bicciato S, Cordenonsi M, Piccolo S. Role of TAZ as mediator of Wnt signaling. Cell. 2012;151(7):1443-56.

13. Cotton JL, Li Q, Ma L, Park JS, Wang J, Ou J, Zhu LJ, Ip YT, Johnson RL, Mao J. YAP/TAZ and Hedgehog Coordinate Growth and Patterning in Gastrointestinal Mesenchyme. Dev Cell. 2017;43(1):35-47 e34.

14. Mussell A, Shen H, Chen Y, Mastri M, Eng KH, Bshara W, Frangou C, Zhang J. USP1 Regulates TAZ Protein Stability Through Ubiquitin Modifications in Breast Cancer. Cancers (Basel) 2020, 12(11).

15. Choi W, Kim J, Park J, Lee DH, Hwang D, Kim JH, Ashktorab H, Smoot D, Kim SY, Choi C, et al. YAP/TAZ Initiates Gastric Tumorigenesis via Upregulation of MYC. Cancer Res. 2018;78(12):330620. 
16. Chen G, Xie J, Huang P, Yang Z. Overexpression of TAZ promotes cell proliferation, migration and epithelial-mesenchymal transition in ovarian cancer. Oncol Lett. 2016;12(3):1821-5.

17. Reyes-Turcu FE, Ventii KH, Wilkinson KD. Regulation and cellular roles of ubiquitin-specific deubiquitinating enzymes. Annu Rev Biochem. 2009;78:363-97.

18. Leznicki $P$, Kulathu Y. Mechanisms of regulation and diversification of deubiquitylating enzyme function. J Cell Sci. 2017;130(12):1997-2006.

19. Ciechanover A, Schwartz AL. The ubiquitin system: pathogenesis of human diseases and drug targeting. Biochim Biophys Acta. 2004;1695(1-3):3-17.

20. Liu Y, Su P, Zhao W, Li X, Yang X, Fan J, Yang H, Yan C, Mao L, Ding Y, et al. ZNF213 negatively controls triple negative breast cancer progression via Hippo/YAP signaling. Cancer Sci. 2021;112(7):2714-27.

21. Zhou X, Li Y, Wang W, Wang S, Hou J, Zhang A, Lv B, Gao C, Yan Z, Pang D, et al: Regulation of Hippo/YAP signaling and Esophageal Squamous Carcinoma progression by an E3 ubiquitin ligase PARK2. Theranostics 2020, 10(21):9443-9457.

22. Zhou R, Ding Y, Xue M, Xiong B, Zhuang T. RNF181 modulates Hippo signaling and triple negative breast cancer progression. Cancer Cell Int. 2020;20:291.

23. Zhang A, Wang W, Chen Z, Pang D, Zhou X, Lu K, Hou J, Wang S, Gao C, Lv B, et al. SHARPIN Inhibits Esophageal Squamous Cell Carcinoma Progression by Modulating Hippo Signaling. Neoplasia. 2020;22(2):76-85.

24. Wang Z, Kong Q, Su P, Duan M, Xue M, Li X, Tang J, Gao Z, Wang B, Li Z, et al. Regulation of Hippo signaling and triple negative breast cancer progression by an ubiquitin ligase RNF187. Oncogenesis. 2020;9(3):36.

25. Ma B, Chen Y, Chen L, Cheng H, Mu C, Li J, Gao R, Zhou C, Cao L, Liu J, et al. Hypoxia regulates Hippo signalling through the SIAH2 ubiquitin E3 ligase. Nat Cell Biol. 2015;17(1):95-103.

26. Zhao B, Tumaneng K, Guan KL. The Hippo pathway in organ size control, tissue regeneration and stem cell self-renewal. Nat Cell Biol. 2011;13(8):877-83.

27. Tang Y, Fang G, Guo F, Zhang H, Chen X, An L, Chen M, Zhou L, Wang W, Ye T, et al: Selective Inhibition of STRN3-Containing PP2A Phosphatase Restores Hippo Tumor-Suppressor Activity in Gastric Cancer. Cancer Cell 2020, 38(1):115-128 e119.

28. Huang C, Yuan W, Lai C, Zhong S, Yang C, Wang R, Mao L, Chen Z, Chen Z. EphA2-to-YAP pathway drives gastric cancer growth and therapy resistance. Int J Cancer. 2020;146(7):1937-49.

29. Jiao S, Guan J, Chen M, Wang W, Li C, Wang Y, Cheng Y, Zhou Z. Targeting IRF3 as a YAP agonist therapy against gastric cancer. J Exp Med. 2018;215(2):699-718.

30. Jiao S, Wang H, Shi Z, Dong A, Zhang W, Song X, He F, Wang Y, Zhang Z, Wang W, et al. A peptide mimicking VGLL4 function acts as a YAP antagonist therapy against gastric cancer. Cancer Cell. 2014;25(2):166-80. 
31. Yin F, Dong J, Kang LI, Liu X. Hippo-YAP signaling in digestive system tumors. Am J Cancer Res. 2021;11(6):2495-507.

32. Gao YB, Chen ZL, Li JG, Hu XD, Shi XJ, Sun ZM, Zhang F, Zhao ZR, Li ZT, Liu ZY, et al. Genetic landscape of esophageal squamous cell carcinoma. Nat Genet. 2014;46(10):1097-102.

33. Kanai F, Marignani PA, Sarbassova D, Yagi R, Hall RA, Donowitz M, Hisaminato A, Fujiwara T, Ito $Y$, Cantley LC, et al. TAZ: a novel transcriptional co-activator regulated by interactions with 14-3-3 and PDZ domain proteins. EMBO J. 2000;19(24):6778-91.

34. Chen YA, Lu CY, Cheng TY, Pan SH, Chen HF, Chang NS. WW Domain-Containing Proteins YAP and TAZ in the Hippo Pathway as Key Regulators in Stemness Maintenance, Tissue Homeostasis, and Tumorigenesis. Front Oncol. 2019;9:60.

35. Nijman SM, Luna-Vargas MP, Velds A, Brummelkamp TR, Dirac AM, Sixma TK, Bernards R. A genomic and functional inventory of deubiquitinating enzymes. Cell. 2005;123(5):773-86.

36. Melo-Cardenas J, Zhang Y, Zhang DD, Fang D. Ubiquitin-specific peptidase 22 functions and its involvement in disease. Oncotarget. 2016;7(28):44848-56.

37. Sun XX, He X, Yin L, Komada M, Sears RC, Dai MS. The nucleolar ubiquitin-specific protease USP36 deubiquitinates and stabilizes c-Myc. Proc Natl Acad Sci U S A. 2015;112(12):3734-9.

38. Fraile JM, Campos-Iglesias D, Rodriguez F, Astudillo A, Vilarrasa-Blasi R, Verdaguer-Dot N, Prado MA, Paulo JA, Gygi SP, Martin-Subero JI, et al. Loss of the deubiquitinase USP36 destabilizes the RNA helicase DHX33 and causes preimplantation lethality in mice. J Biol Chem. 2018;293(6):2183-94.

39. Taillebourg E, Gregoire I, Viargues P, Jacomin AC, Thevenon D, Faure M, Fauvarque MO. The deubiquitinating enzyme USP36 controls selective autophagy activation by ubiquitinated proteins. Autophagy. 2012;8(5):767-79.

\section{Figures}

\section{Figure 1}

\section{DUB1 is elevated in human gastric cancer and correlates with Hippo signaling activity in whole genomic scale}

A: The siRNA screening flow process to identify novel deubiquitinase in modulating Hippo signaling. HEK293 cells were seeded in 96-well plate. For each well, one kind of deubiquitinase siRNA were transfected and incubated for 48 hours. The cells were subject to RNA extraction and QPCR analysis for CTGF expression.

B: The relative mRNA level of CTGF by the deubiquitinase siRNA screening. The green dots represented the DUBs already reported to link to Hippo signaling in previous study. The red dot represented the CTGF mRNA level by DUB1 depletion. 
C: DUB1 expression level was significantly elevated in different stages of gastric cancer compared with normal gastric tissue. Data were generated from TCGA database (https://www.genome.gov/).

D: DUB1 protein expression was significantly increased in gastric tumor tissues compared with their adjacent non-tumor tissues as analyzed by immunohistochemistry.

E: The correlation between DUB1 expression and clinical-pathological characteristics in gastric tumor samples. The data analysis revealed that DUB1 expression correlated with lymph node metastasis and advanced tumor stage $(P=0.0002 ; P<0.0001$ respectively $)$.

F: DUB1 expression was significantly correlated with increased TAZ levels in gastric cancer specimens analyzed by $\mathrm{IHC}$ staining.

G: Kaplan-Meier analysis revealed that DUB1 expression was related with poorer progression-free survival of gastric cancer patients. $P<0.001$, log-rank test.

$\mathrm{H}$ : Kaplan-Meier analysis revealed that TAZ expression was related with poorer progression-free survival of gastric cancer patients. $P=0.0016$, log-rank test.

I: Top 10 KEGG pathways significantly decreased (top) or increased (bottom) in MCG803 cells treated with siDUB1. The pathway-enrichment analysis was used by the threshold $P<0.001$ and fold change $>2$ to derive regulated genes. The cells were treated with vehicle or $50 \mathrm{nM}$ siDUB1 for 48 hours. The whole mRNA was extracted for RNA sequence analysis. $n=3$.

J: Gene set enrichment analysis (GSEA) showed a depletion of Hippo pathway signature genes in MGC803 cells treated with DUB1 siRNA.

K: Volcano plot showed the DUB1 depletion could inhibit the Hippo pathway signature genes (red) in MCG823 cells. Threshold $P<0.05$ and Fold change $>1.5$. 


\section{Figure 2}

A

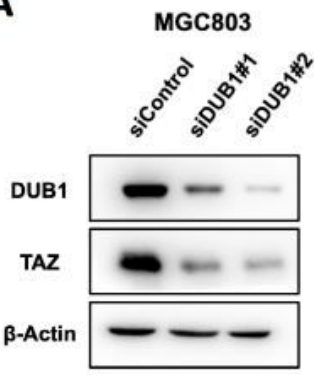

B

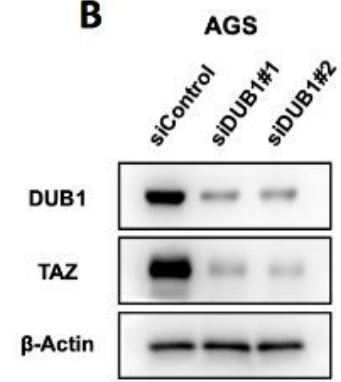

C

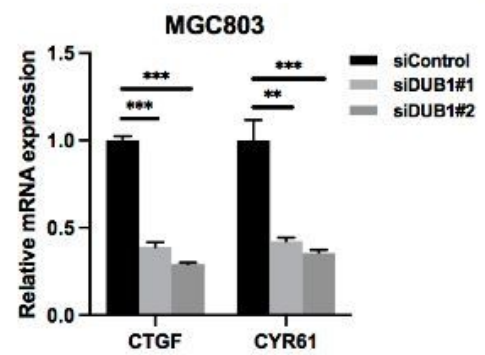

D

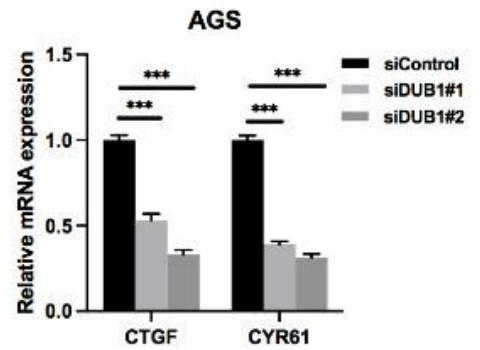

E

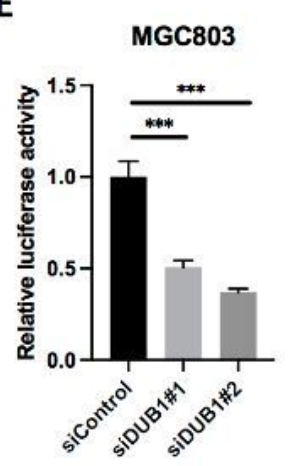

F

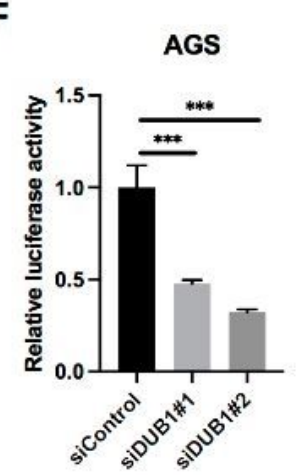

G

HEK-293T

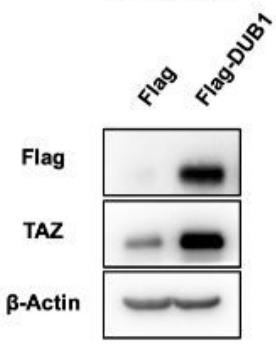

H

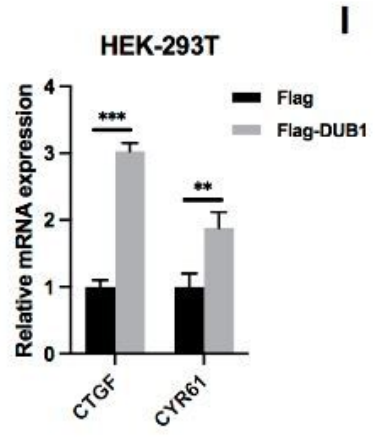

HEK-293T

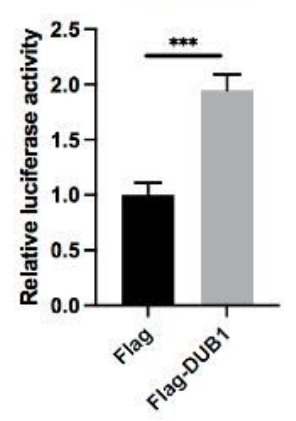

Figure 2

\section{DUB1 facilitates Hippo/TAZ axis in gastric cancer cells}

A: DUB1 depletion inhibited TAZ protein levels in MGC803 cells. MGC803 cells were transfected with siControl or siDUB1. After $48 \mathrm{~h}$, cells were harvested for western blot analysis. DUB1 and TAZ protein levels were determined by Western blot. Actin was used as internal control.

B: DUB1 depletion inhibited TAZ protein levels in AGS cells. AGS cells were transfected with siControl or siDUB1. After $48 \mathrm{~h}$, cells were harvested for western blot analysis. DUB1 and TAZ protein levels were determined by Western blot. Actin was used as internal control.

C: DUB1 depletion decreased Hippo target gene expression in MGC803 cells. MGC803 cells were transfected with siControl or siDUB1. After $48 \mathrm{~h}$, total RNA was extracted for gene expression analysis. Each group was done in triplicates. ${ }^{*} P<0.05 ;{ }^{\star \star} P<0.01 ;{ }^{* \star *} P<0.001$ for target gene expression comparison.

D: DUB1 depletion decreased Hippo target gene expression in AGS cells. AGS cells were transfected with siControl or siDUB1. After $48 \mathrm{~h}$, total RNA was extracted for gene expression analysis. Each group was done in triplicates. ${ }^{\star} \mathrm{P}<0.05 ;{ }^{*} \mathrm{P}<0.01 ;{ }^{* \star *} \mathrm{P}<0.001$ for target gene expression comparison.

E: DUB1 depletion decreased TEAD Luciferase activity in MGC803 cells. MGC803 cells were transfected with siControl or siDUB1. After $24 \mathrm{~h}$, cells were transfected with TEAD luciferase reporter plasmids. After $24 \mathrm{~h}$, cells harvested for luciferase activity analysis. 
F: DUB1 depletion decreased TEAD Luciferase activity in AGS cells. AGS cells were transfected with siControl or siDUB1. After $24 \mathrm{~h}$, cells were transfected with TEAD luciferase reporter plasmids. After $24 \mathrm{~h}$, cells harvested for luciferase activity analysis.

G: DUB1 over-expression increased TAZ protein levels in HEK293 cells. HEK293 cells were transfected with 2 ug Flag-DUB1 or Flag vector. After 48 h, cells were harvested for western blot analysis. DUB1 and TAZ protein levels were determined by Western blot. Actin was used as internal control.

H: DUB1 overexpression increased Hippo target gene expression in HEK293 cells. HEK293 cells were transfected with 2 ug Flag-DUB1 or Flag vector. After $48 \mathrm{~h}$, total RNA was extracted for gene expression analysis. Each group was done in triplicates. ${ }^{*} \mathrm{P}<0.05$; ${ }^{*} \mathrm{P}<0.01 ;{ }^{* \star *} \mathrm{P}<0.001$ for target gene expression comparison.

I: DUB1 overexpression increased TEAD Luciferase activity in HEK293 cells. HEK293 cells were transfected with 2 ug Flag-DUB1 or Flag vector. After 24 h, cells were transfected with TEAD luciferase reporter plasmids. After $24 \mathrm{~h}$, cells harvested for luciferase activity analysis. 
Figure 3
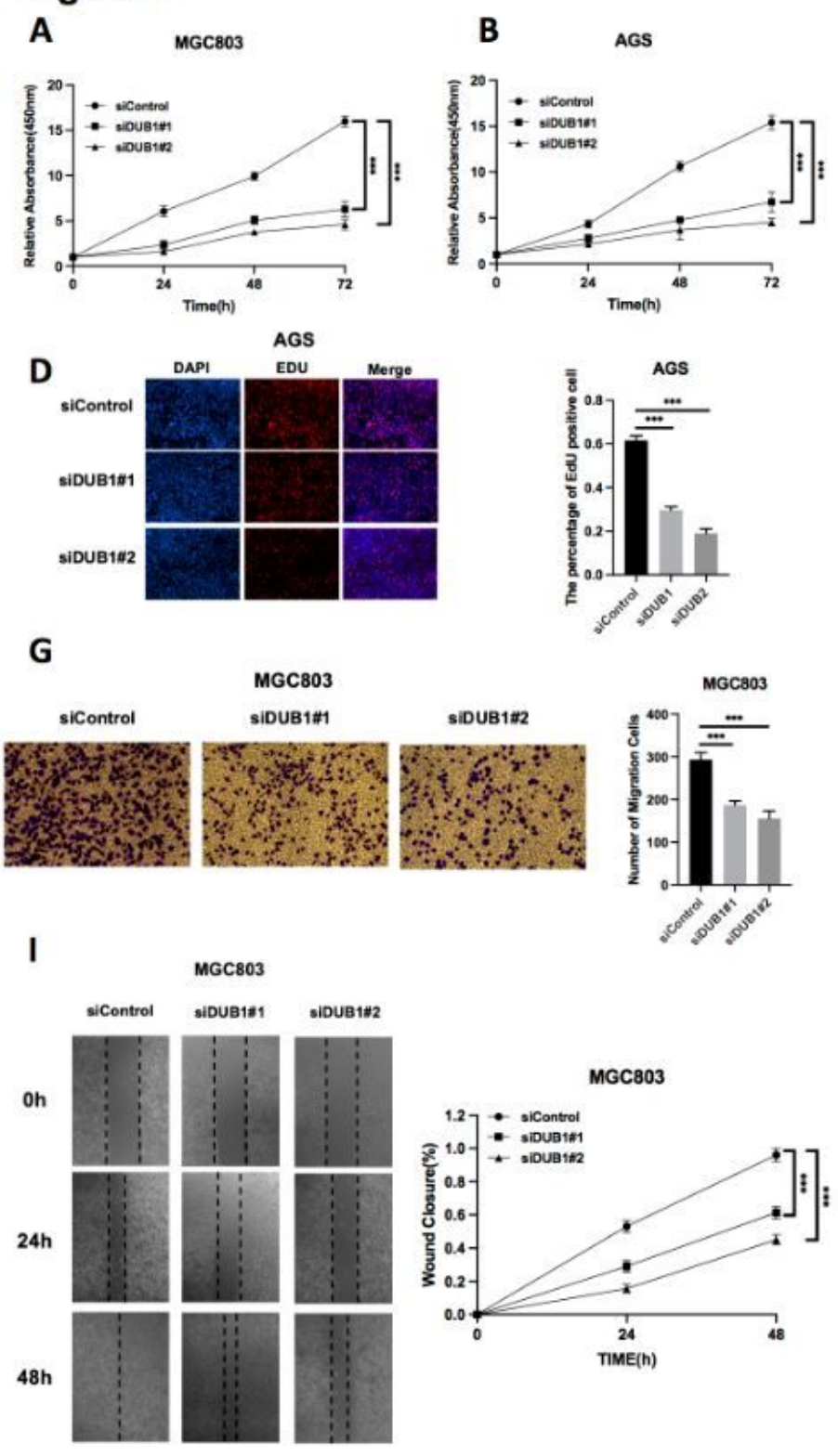

K

$\mathbf{L}$
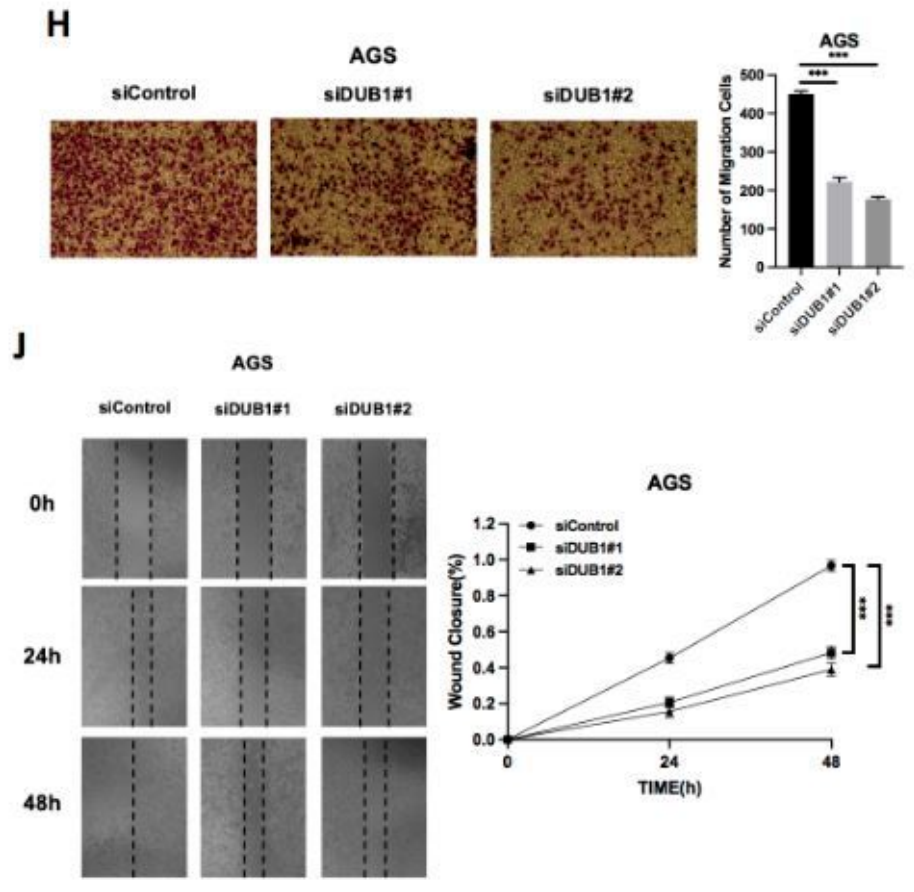

M

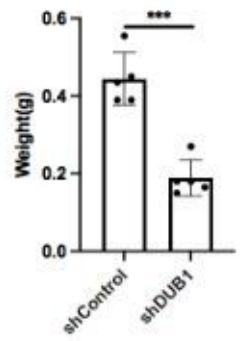

Figure 3

DUB1 depletion inhibits gastric cancer progression in vivo and in vitro.

A-B: Depletion of DUB1 inhibited the proliferation of gastric cancer cells. MGC803 and AGS cells were transfected with siControl or siDUB1. There were two different siRNA be used. After 24 hours, the assay of 
WST-1 was used to determine the cellar metabolic activity at indicated time points after infection. Experiments were done in triplicates. ${ }^{\star} P<0.05$; ${ }^{\star \star} P<0.01 ;{ }^{\star \star \star} P<0.001$ for cell growth comparison.

C-D: DUB1 depletion inhibited the number of EdU positive gastric cancer cells. MGC803 and AGS cells were transfected with siControl or siDUB1. After 24 hours, EdU was added into the medium for 2 hours incubation. The absolute cell number was counted to indicate cell proliferation activity.

E-F: Cell cycle analysis to assess the effect of DUB1 silencing in MGC803 cells. MGC803 cells were transfected with $50 \mathrm{nM}$ DUB1 siRNA or $50 \mathrm{nM}$ control siRNA. After 24 hours, the cells were harvested, fixed with $70 \%$ ethanol and stained with propidium iodide. The cells were subjected to FACS analysis. Experiments were performed in triplicate. ${ }^{*} \mathrm{P}<0.05$; ${ }^{* *} \mathrm{P}<0.01$; ${ }^{* \star *} \mathrm{P}<0.001$ for cell proportion comparisons. Representative histograms and cell cycle phase distributions are shown in Figure 3E and 3F, respectively.

G-H: DUB1 promoted gastric cancer cell migration in MGC803 and AGS cells. MGC803 and AGS cells were transfected with siControl or siDUB1. After 24 hours transwell was used to check the migration capacity. The cell number was counted and Data are presented as $\pm S D$. ${ }^{\star *} P<0.01$, ${ }^{\star \star *} P<0.001$ (student's ttest).

I-J: Wound-healing assay of MGC803 and AGS cells with DUB1 depletion or siControl. Quantification of wound closure at the indicated time points. Data are presented as \pm SD. ${ }^{\star *} \mathrm{P}<0.01,{ }^{\star \star *} \mathrm{P}<0.001$ (student's ttest).

K-M: DUB1 depletion inhibited gastric tumor growth in vivo. MGC803 cells were stably transduced with lentiviral vectors carrying scrambled shRNA or DUB1 shRNA. MGC803 cells $\left(2 \times 10^{6}\right)$ were injected into the right dorsal flank of 4-week-old female BALB/c nude mice. Tumor formation in nude mice was monitored over a 4-week period. The tumor volume was calculated by the formula: tumor volume $=0.5 \times$ length $\times$ width $^{2}$. Mice were sacrificed five weeks after tumor cell injection. Tumor growth curves, weights and photographs are shown in panels $K, L$ and $M$, respectively. 


\section{Figure 4}

\section{A}

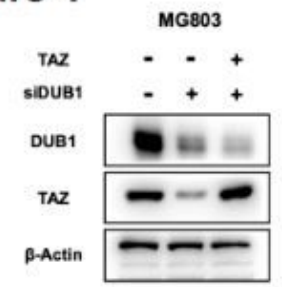

$\mathbf{E}$
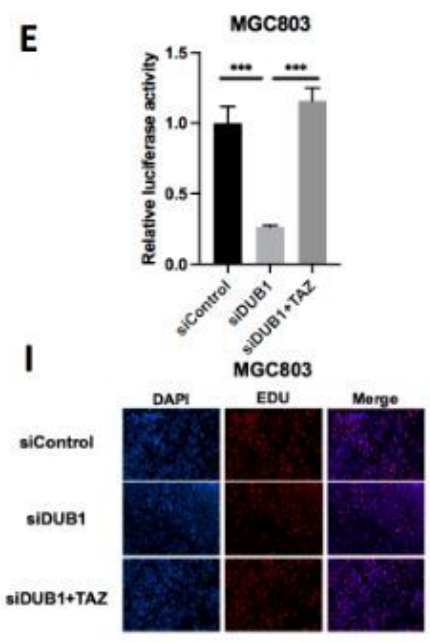

$\mathbf{K}$

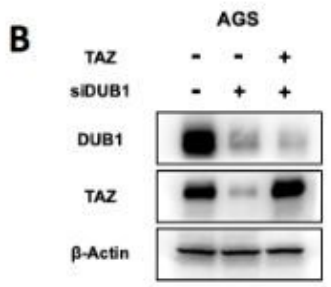

F
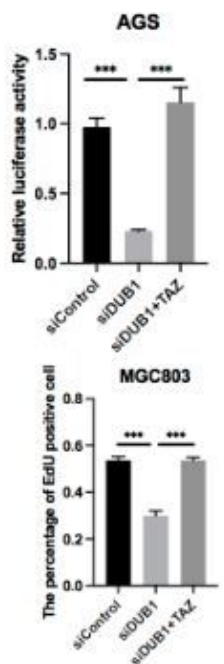

$\mathbf{L}$
C

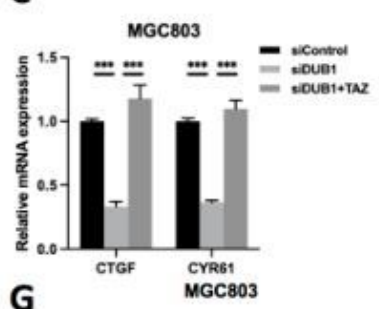

G

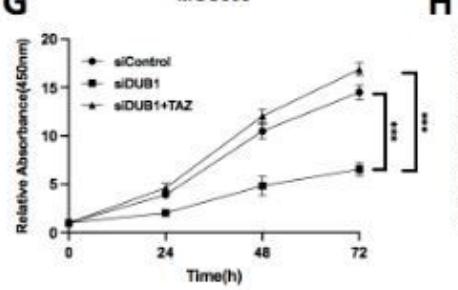

H
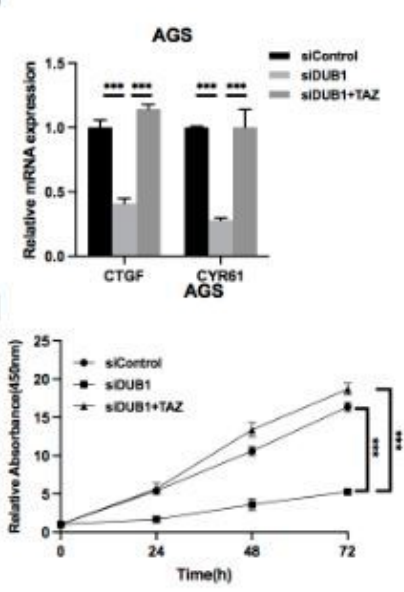

J
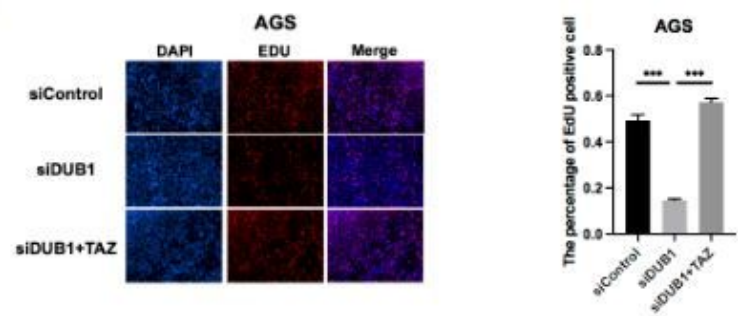

M

\section{Figure 4}

DUB1 controls gastric cancer progression via Hippo/TAZ axis

A: DUB1 depletion decreased TAZ protein level, which effect could be reversed by TAZ knocking-down. MGC803 cells were transfected with siControl or siDUB1. After $24 \mathrm{~h}$, cells were transfected with siTAZ or 
siControl. After $48 \mathrm{~h}$, cells were harvested for western blot analysis. DUB1 and TAZ protein levels were determined by Western blot. Actin was used as internal control.

B: DUB1 depletion decreased TAZ protein level, which effect could be reversed by TAZ knocking-down. AGS cells were transfected with siControl or siDUB1. After $24 \mathrm{~h}$, cells were transfected with siTAZ or siControl. After $48 \mathrm{~h}$, cells were harvested for western blot analysis. DUB1 and TAZ protein levels were determined by Western blot. Actin was used as internal control.

C: DUB1 depletion suppressed Hippo target gene expression, which effect could be reversed by TAZ overexpression. MGC803 cells were transfected with siControl or siDUB1. After $24 \mathrm{~h}$, cells were transfected with Flag-TAZ or Flag vector. After $48 \mathrm{~h}$, total RNA was extracted for gene expression analysis. Each group was done in triplicates. ${ }^{*} P<0.05 ;{ }^{*} P<0.01 ; * \star \star P<0.001$ for target gene expression comparison.

D: DUB1 depletion suppressed Hippo target gene expression, which effect could be reversed by TAZ overexpression. AGS cells were transfected with siControl or siDUB1. After $24 \mathrm{~h}$, cells were transfected with Flag-TAZ or Flag vector. After $48 \mathrm{~h}$, total RNA was extracted for gene expression analysis. Each group was done in triplicates. ${ }^{*}<<0.05 ;{ }^{*} \mathrm{P}<0.01 ; * \star * P<0.001$ for target gene expression comparison.

E: DUB1 depletion decreased TEAD Luciferase activity in MGC803 cells, which effect could be reversed by TAZ overexpression. MGC803 cells were transfected with siControl or siDUB1. After $24 \mathrm{~h}$, cells were transfected with Flag-TAZ or Flag vector. After $24 \mathrm{~h}$, cells were transfected with TEAD luciferase reporter plasmids. The cells harvested for luciferase activity analysis.

F: DUB1 depletion decreased TEAD Luciferase activity in AGS cells, which effect could be reversed by TAZ overexpression. AGS cells were transfected with siControl or siDUB1. After $24 \mathrm{~h}$, cells were transfected with Flag-TAZ or Flag vector. After $24 \mathrm{~h}$, cells were transfected with TEAD luciferase reporter plasmids. The cells harvested for luciferase activity analysis.

G: Cell growth inhibition induced by DUB1 silencing was rescued by TAZ overexpression in MGC803 cells. MGC803 cells were transfected with $50 \mathrm{nM}$ DUB1 siRNA or $50 \mathrm{nM}$ control siRNA. After $24 \mathrm{~h}$, cells were transfected with Flag-TAZ or Flag vector. After 24 hours, a CCK-8 assay was used to determine the cellular metabolic activity at the indicated time points after transfection. Experiments were performed in triplicate. ${ }^{*} \mathrm{P}<0.05 ; * \star \mathrm{P}<0.01 ; * \star \star \mathrm{P}<0.001$ for cell growth comparisons.

$\mathrm{H}$ : Cell growth inhibition induced by DUB1 silencing was rescued by TAZ overexpression in AGS cells. AGS cells were transfected with $50 \mathrm{nM}$ DUB1 siRNA or $50 \mathrm{nM}$ control siRNA. After $24 \mathrm{~h}$, cells were transfected with Flag-TAZ or Flag vector. After 24 hours, a CCK-8 assay was used to determine the cellular metabolic activity at the indicated time points after transfection. Experiments were performed in triplicate. ${ }^{*} \mathrm{P}<0.05 ;{ }^{* \star} \mathrm{P}<0.01 ; * \star * \mathrm{P}<0.001$ for cell growth comparisons.

I-J: DUB1 depletion inhibited the number of EdU positive gastric cancer cells. Which effect could be further rescued by TAZ overexpression. MGC803 and AGS cells were transfected with siControl or siDUB1. 
After $24 \mathrm{~h}$, cells were transfected with Flag-TAZ or Flag vector. The EdU was added into the medium for 2 hours incubation. The absolute cell number was counted to indicate cell proliferation activity.

K-L: Cell cycle arrest caused by DUB1 silencing was partially rescued by TAZ overexpression in MGC803 cells. MGC803 cells were transfected with $50 \mathrm{nM}$ DUB1 siRNA or $50 \mathrm{nM}$ control siRNA. After $24 \mathrm{~h}$, cells were transfected with Flag-TAZ or Flag vector. The cells were harvested, fixed with $70 \%$ ethanol and stained with propidium iodide. The cells were subjected to FACS analysis. Experiments were performed in triplicate. ${ }^{*} \mathrm{P}<0.05 ;{ }^{*} \mathrm{P}<0.01 ;{ }^{* \star *} \mathrm{P}<0.001$ for cell proportion comparisons. Representative histograms and cell cycle phase distributions are shown in Figure $4 \mathrm{~K}$ and $4 \mathrm{~L}$, respectively.

M: The clone formation capacity inhibited by DUB1 silencing could be rescued by TAZ overexpression in MGC803 and AGS cells. The gastric cancer cells were transfected with $50 \mathrm{nM}$ DUB1 siRNA or $50 \mathrm{nM}$ control siRNA. After $24 \mathrm{~h}$, cells were transfected with Flag-TAZ or Flag vector. Quantification of clone formation was shown at the indicated time points. Data are presented as $\pm S D$. ${ }^{\star \star}, P<0.01, \star \star \star, P<0.001$ (student's t-test).

N-0: DUB1 depletion decreased gastric cell invasion capacity, which effect could be reversed by TAZ overexpression. MGC803 and AGS cells were transfected with $50 \mathrm{nM}$ siControl or siDUB1. After $24 \mathrm{~h}$, cells were transfected with Flag-TAZ or Flag vector. After another $24 \mathrm{~h}$, cancer cells were seeded into the chamber for trans-well assay. The cell number was counted and Data are presented as $\pm S D$. ${ }^{\star *} P<0.01$, $\star \star \star * P<0.001$ (student's t-test).

P-Q: DUB1 depletion decreased gastric cell migration capacity, which effect could be reversed by TAZ overexpression. MGC803 and AGS cells were transfected with $50 \mathrm{nM}$ siControl or siDUB1. After $24 \mathrm{~h}$, cells were transfected with Flag-TAZ or Flag vector. After another $24 \mathrm{~h}$, the cells were seeded in a 6 -well plate until confluent and then wounded with a sterile tip. The cells pictures were acquired at the indicated time points after scratching. 
Figure 5

A

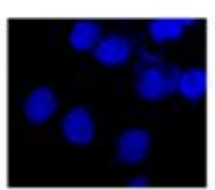

DAPI

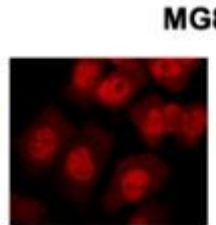

TAZ
MG803

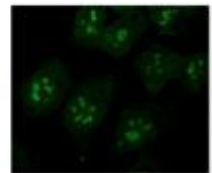

DUB1

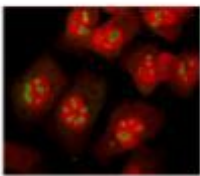

Merge
B

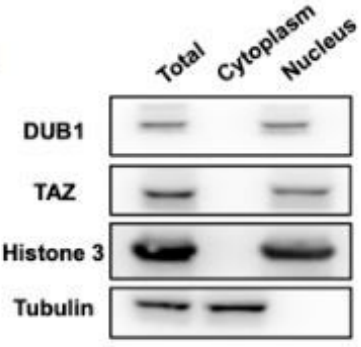

C

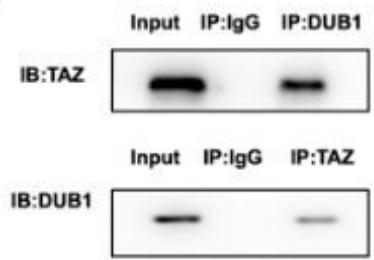

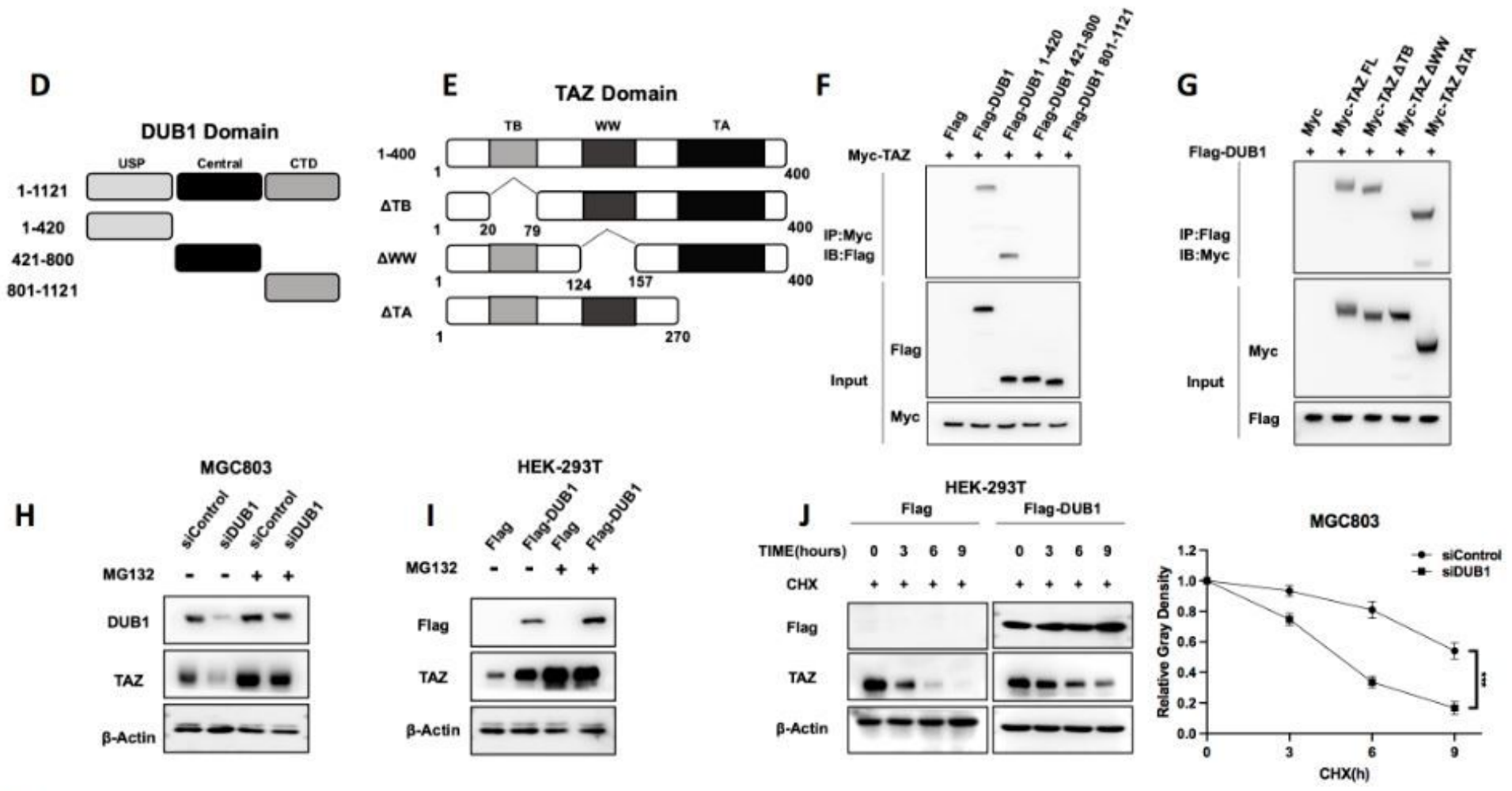

K

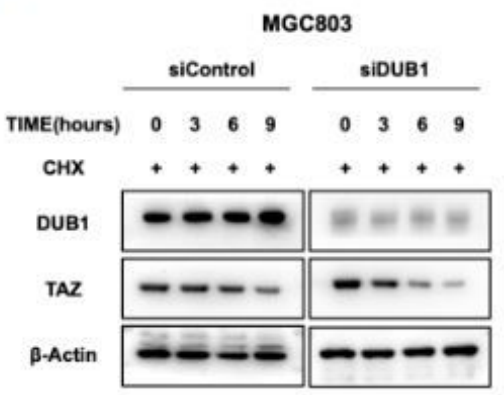

HEK-293T

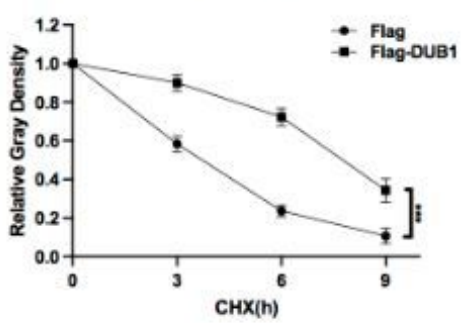

Figure 5

DUB1 associates with TAZ and modulates TAZ stability in gastric cancer cells

A: Intracellular localization analysis of DUB1 and TAZ by immunofluorescence assay. MGC803 cells were cultured in normal medium before fixation. Intracellular localization of DUB1 (green) and TAZ (red) is shown. Nuclei (blue) were stained with 4',6-diamidino-2-phenylindole (DAPI). 
B: DUB1 and TAZ were mainly localized in the nuclear in MGC803 cells. The subcellular protein fractionation kit (Thermo scientific, 78840) was used for cytoplasm and nuclear separation. Tubulin and Histone-3 were used for cytoplasm and nuclear control.

C: Co-IP assay revealed an association between endogenous DUB1 and TAZ in MGC803 cells. MGC803 cells were harvested with RIPA lysis buffer. Co-IP was performed using antibody as indicated.

D-E: DUB1 domain structure and deletion mutants were used in the study (full length, USP domain, Central domain and CTD domain). TAZ full-length and deletion mutants are used in the study (full length, $\triangle T$ TA domain, $\triangle \mathrm{WW}$ domain, $\triangle \mathrm{TBD}$ domain).

F: DUB1 interacted with TAZ through its USP domain. HEK293 cells were transfected with $2 \mu \mathrm{g}$ Myc-TAZ together with Flag-DUB1 full length or mutants (full length, USP domain, Central domain and CTD domain). After $24 \mathrm{~h}$, cells were harvested with NP-40 lysis buffer. Co-IP was performed using Myc antibody. The possible interacted DUB1 domains were detected by Flag antibody.

G: TAZ interacted with DUB1 through its WW domain. HEK293 cells were transfected with $2 \mu \mathrm{g}$ Flag-DUB1 together with Myc-TAZ full length or mutants (full length, $\triangle T A$ domain, $\triangle W W$ domain, $\triangle T B D$ domain). After $24 \mathrm{~h}$, cells were harvested with NP-40 lysis buffer. Co-IP was performed using Flag antibody. The possible interacted DUB1 domains were detected by Flag antibody.

$\mathrm{H}$ : In the presence of the proteasome inhibitor MG132, the effect of DUB1 on TAZ did not further increase TAZ protein levels. MGC803 cells were transfected $50 \mathrm{nM}$ siDUB1 or siControl. After $24 \mathrm{~h}$, cells were treated with $10 \mathrm{mM} \mathrm{MG132/vehicle} \mathrm{for} 6 \mathrm{~h}$. Cell lysates were prepared for Western blot analysis. The results are representative for three independent experiments.

I: In the presence of the proteasome inhibitor MG132, the stabilization effect of DUB1 on TAZ did not further increase TAZ protein levels. HEK293 cells were transfected with $0.5 \mathrm{ug}$ Flag or Flag -DUB1 plasmids. After $24 \mathrm{~h}$, cells were treated with $10 \mathrm{mM} \mathrm{MG132/vehicle} \mathrm{for} 6 \mathrm{~h}$. Cell lysates were prepared for Western blot analysis. The results are representative for three independent experiments.

J: DUB1 overexpression increased TAZ half-life in HEK293 cells. HEK293 cells were transfected with 0.5 ug Flag-tag or Flag-DUB1 plasmids. After $24 \mathrm{~h}$, cells were treated with $100 \mathrm{mM}$ cycloheximide/vehicle for indicated times. Cell lysates were prepared for Western blot analysis. The results are representative for three independent experiments. The TAZ relative density was measured by Image $\mathrm{J}$ software.

K: DUB1 depletion decreased TAZ half-life in MGC803 cells. MGC803 cells were transfected with $50 \mathrm{nM}$ siControl or siDUB1. After $24 \mathrm{~h}$, cells were treated with $100 \mathrm{mM}$ cycloheximide/vehicle for indicated times. Cell lysates were prepared for Western blot analysis. The results are representative for three independent experiments. The TAZ relative density was measured by Image $\mathrm{J}$ software. 


\section{Figure 6}

\section{DUB1 stabilizes TAZ via inhibiting TAZ K48-linked poly-ubiquitination.}

A: DUB1 decreases poly-ubiquitination of TAZ. HEK293 cells were transfected with 2 ug TAZ plasmid, 0.5 ug HA Ub plasmid and $0.5 \mu \mathrm{g}$ Flag-tag or Flag-DUB1 plasmids. The cell extracts were immunoprecipitated with Myc antibody. The poly-ubiquitinated TAZ was detected via western blotting analysis.

B: DUB1 decreases K48-linked poly-ubiquitination of TAZ. HEK293 cells were transfected with 2 ug TAZ plasmid, 0.5 ug HA-K48-Ubi plasmid and $0.5 \mu \mathrm{g}$ Flag-tag or Flag-DUB1 plasmids. The cell extracts were immunoprecipitated with Myc antibody. The K48-linked poly-ubiquitinated TAZ was detected via western blotting analysis.

C: DUB1 depletion promotes the poly-ubiquitination of TAZ in MGC803 cells. MGC803 cells were transfected with $50 \mathrm{nM}$ siDUB1 or siControl. After 24 hours, cells were transfected with 2 ug TAZ plasmid and $0.5 \mathrm{ug} \mathrm{HA} \mathrm{Ub}$ plasmid. The cell extracts were immunoprecipitated with Myc antibody. The polyubiquitinated TAZ was detected via western blotting analysis.

D: DUB1 depletion promotes K48-linked poly-ubiquitination of TAZ in MGC803 cells. MGC803 cells were transfected with $50 \mathrm{nM}$ siDUB1 or siControl. After 24 hours, cells were transfected with 2 ug TAZ plasmid and 0.5 ug HA-K48 Ubi plasmid. The cell extracts were immunoprecipitated with Myc antibody. The K48linked poly-ubiquitinated TAZ was detected via western blotting analysis.

EXThe USP domain of DUB1 is required for it to inhibit poly-ubiquitination on TAZ. HEK293 cells were transfected with $2 \mu \mathrm{g}$ Myc-TAZ, $0.5 \mu \mathrm{g}$ HA Ub plasmid and $0.5 \mu \mathrm{g}$ Flag-DUB1 full length or mutants (USP domain, Central domain and CTD domain). The polyubiquitinated TAZ was detected via western blotting analysis.

F: The USP domain of DUB1 is required for it to inhibit K48-linked poly-ubiquitination on TAZ. HEK293 cells were transfected with $2 \mu \mathrm{g}$ Myc-TAZ, $0.5 \mu \mathrm{g} \mathrm{HA} \mathrm{K48-Ubi} \mathrm{plasmid} \mathrm{and} 0.5 \mu \mathrm{g}$ Flag-DUB1 full length or mutants (USP domain, Central domain and CTD domain). The K48-linked polyubiquitinated TAZ was detected via western blotting analysis.

G: The hypothetical model for DUB1 regulating Hippo/TAZ axis in gastric cancer. DUB1 protein associated with TAZ and enhanced TAZ protein stability via inhibiting TAZ K48-linked poly-ubiquitination, which facilitated the activation of Hippo/TAZ axis and gastric cancer progression.

\section{Supplementary Files}

This is a list of supplementary files associated with this preprint. Click to download.

- Originaldata.xlsx

- celllineauthenticationDUB1.pdf 
- siDUBscreening.xlsx

- wbfilms.pptx 